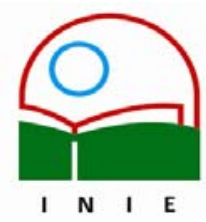

Actualidades Investigativas en Educación

Revista Electrónica publicada por el

Instituto de Investigación en Educación

Universidad de Costa Rica

ISSN 1409-4703

http://revista.inie.ucr.ac.cr

COSTA RICA

\title{
LA OPINIÓN DE UN GRUPO DE DOCENTES SOBRE LA DESERCIÓN ESCOLAR. EXPLORANDO SOBRE SUS ACTUACIONES EN EL CONTEXTO INSTITUCIONAL
}

THE OPINION OF A GROUP OF TEACHERS ABOUT SCHOOL DESERTION: EXPLORING THEIR ACTS IN THE INSTITUTIONAL CONTEXT

\author{
Volumen 8 , Número 3 \\ pp. 1-33
}

Este número se publicó el 15 de diciembre 2008

Teresita Cordero Cordero

La revista está indexada en los directorios:

LATINDEX, REDALYC, IRESIE, CLASE, DIALNET, DOAJ, E-REVIST@S,

La revista está incluida en los sitios:

REDIE, RINACE, OEI, MAESTROTECA, HUASCARAN

Los contenidos de este artículo están bajo una licencia Creative Commons 


\title{
LA OPINIÓN DE UN GRUPO DE DOCENTES SOBRE LA DESERCIÓN ESCOLAR. EXPLORANDO SOBRE SUS ACTUACIONES EN EL CONTEXTO INSTITUCIONAL
}

\author{
THE OPINION OF A GROUP OF TEACHERS ABOUT SCHOOL DESERTION: EXPLORING \\ THEIR ACTS IN THE INSTITUTIONAL CONTEXT
}

Teresita Cordero Cordero ${ }^{1}$

\begin{abstract}
Resumen: El trabajo indagó con un grupo de docentes de cinco escuelas y un colegio costarricenses las acciones que suelen hacer ante la deserción escolar. Desde una perspectiva de investigación de corte cualitativo se organiza la información y se retoman estudios para presentar la problemática en el país. Se argumenta que la deserción es un problema multicausal, donde la organización de los centros educativos y las acciones que realiza el personal docente de las instituciones también viene contribuir con el problema.

El estudio plantea en términos generales que como país tenemos una legislación avanzada para la niñez y la adolescencia pero no hay una política clara sobre qué hacer en los casos de deserción. Si bien solamente se trabajó en un colegio parece existir una mejor respuesta en la primaria para retener o mantener al estudiantado. Lograr una organización eficiente de las instituciones escolares con respecto a cómo enfrentar la deserción podría ser una vía para mitigar esta problemática. Tarea que estaría pendiente y que debería ser trabajada desde el Ministerio de Educación Pública costarricense con el objetivo de incidir en la cultura institucional de las diferentes escuelas y colegios del país.
\end{abstract}

Palabras clave: DESERCIÓN ESCOLAR, PERSPECTIVA DOCENTE, CULTURA ORGANIZATIVA ESCOLAR.

Abstract: This work deals with a group of teachers from five schools and one high school in Costa Rica and their actions regarding school desertion. In order to present the current situation of this problem in Costa Rica, this information is organized and studies are restarted from the point of view of qualitative research. It is argued that desertion is a problem with multiple causes, where the organization of the education centers and the actions of the teacher staff compounds this issue.

The study states in general terms that as a country, although we have advanced legislation in favor of children and adolescents, we do not have a clear policy for handling desertion. Despite we only worked with a single high school, we believe that there is a better response in primary school to the efforts at keeping students. Achieving an efficient organization of schools regarding how to deal with desertion could be the way to improve this problem. This is a pending task and it should be addressed by the Ministry of Public Education in Costa Rica, with the objective of making an impact in the institutional culture of the different schools and high schools in the country.

Key words: SCHOOL DESERTION, TEACHER'S PERSPECTIVE, ORGANIZATIONAL CULTURE AT SCHOOLS.

\footnotetext{
Doctora en Educación del Programa Latinoamericano de Educación, Master en Psicología ambos títulos de la Universidad de Costa Rica. Desde 1991 es profesora en la Escuela de Psicología de la UCR, ocupa el puesto de subdirectora en la misma Escuela y es investigadora en el Instituto de Investigación en Educación (INIE) de la UCR. Ha ocupado los puestos de coordinadora de Docencia de la Escuela de Psicología, profesora investigadora en el Instituto de Investigaciones Psicológicas (IIP). Se ha desarrollado en el campo de la Psicología Social Comunitaria y actualmente esta estudiando los temas de desarrollo infantil, estudios culturales $y$ educación.
}

Dirección electrónica: teresita.cordero@ucr.ac.cr

Artículo recibido: 12 de mayo, 2008

Aprobado: 11 de noviembre, 2008 


\section{Introducción}

La educación formal es un sistema complejo y en el mundo moderno se organiza con el objetivo de ser un medio de transmisión de conocimientos, valores, visiones de mundo que forman a las nuevas generaciones. En la actualidad, este sistema está compitiendo ${ }^{2}$ con otras formas de acceso al conocimiento tales como los medios de comunicación social, la Internet, las familias y las comunidades. No obstante, la Escuela sigue siendo altamente valorada como la vía expedita para posibilitar el desarrollo humano en el marco de una sociedad capitalista que demanda trabajadores con competencias más acordes con las necesidades del mundo de laboral. Desde este punto de vista, la deserción escolar cobra suma importancia sobre todo para los sectores de la población en condiciones de mayor vulnerabilidad social (Espínola y León, 2002).

Machado, Palacios, Crotti y García-Huidobro (2005) comentan, con acierto, que el Derecho a la Educación en el siglo XX fue posible por las conquistas de los sectores sociales, que exigieron garantizar el acceso a la escuela, reflejado en leyes de obligatoriedad y gratuidad, y cuya intervención estatal, garantizó una infraestructura escolar física y de personal docentes, así como una permanencia de años mínima en el sistema para el alumnado. Plantean estos autores, que hay en la actualidad un agotamiento de la escolarización obligatoria y la consecuente certificación, lo que está obligando a pensar en otras respuestas complementarias, relacionados con el derecho a aprender ${ }^{3}$. En otras palabras se requieren procesos para que todas las personas, sean niños, niñas, jóvenes y adultos tengamos la oportunidad de contar con una educación de calidad, para desarrollar las potencialidades como seres humanos.

Sin olvidar promover los valores universales de "la igualdad entre las personas, el respeto a la diversidad y la tolerancia, la no discriminación, la promoción del bien común... Se debe equipar a los niños y jóvenes con las capacidades y conocimientos necesarios para asumir competentemente su condición de ciudadanos. Ofrecer oportunidades múltiples y adecuadas a las diferentes necesidades y situaciones de los

\footnotetext{
${ }^{2}$ Tan solo el dato que plantea la primer encuesta sobre el Estado de la Libre Expresión en Costa Rica, la cual indica la importancia del uso de los medios de comunicación en la vida diaria: "Hoy podemos concluir, a partir de esta encuesta, que el primer socializador es la televisión, lo cual implica que los comunicadores y dueños de los medios de televisión tienen una responsabilidad primigenia en la educación, ya no en los que están formados, sino en los que se están formando", Fonseca y Blanco (2008).

3 Se anota en negrita para enfatizar la importancia que tiene el aprendizaje en los seres humanos sin circunscribirlo de manera exclusiva a las formas de titulación oficiales.
} 
alumnos para que todos desarrollen efectivamente dichas capacidades. (Machado et al., 2005, p. 9)

Así los Derechos Humanos como legado social nos obliga a gestar acciones que permitan una educación inclusiva que no solo se ajuste a la realidad de los grupos humanos, sino que sea un medio de protección a la niñez y adolescencia. Bajo este ideal de inclusión social en oposición a la exclusión, se consideró importante conocer que hacen un grupo de docentes en sus actividades cotidianas y como institución educativa ante el tema de la deserción escolar, en el marco del derecho a la educación.

Ahora bien, es importante hacer notar que tal como lo señala Gajardo (2005) algunos de los grandes problemas del sistema educativo formal en América Latina: las diferencias entre la educación pública y privada, la cual muestra la desigualdad; la mala calidad de la educación reflejada, en las tasas de repetición y deserción temprana -de nuestra parte diríamos de exclusión escolar, como una forma de violación al derecho a la Educación-; los bajos puntajes en la pruebas internacionales ${ }^{4}$; el centralismo y escasa autonomía ${ }^{5}$; las malas condiciones de trabajo docente; la enseñanza descontextualizada de la vivencias de las y los alumnos; y el poco y desigual financiamiento por estudiante.

Garbanzo y Orozco (2007) para el caso de Costa Rica anotan otras problemáticas que se unen a las anteriores tales como la universalización del preescolar y la secundaria; fortalecer la evaluación, monitoreo y la investigación educativa.

Dichos problemas son complejos y profundos, se arrastran año con año, y podríamos argumentar que se agudizan con el tiempo. Tal como se puede comprender el sistema educativo formal tiene grandes retos y problemas que enfrentar los cuales deberían tener una correspondiente acción de parte de nuestros gobiernos. En medio del conjunto de problemáticas encontramos la deserción, no como una situación que atañe solo al desertor y su familia, sino al sistema educativo.

\footnotetext{
${ }^{4}$ Tema cuestionable que ameritaría mayor estudio y reflexión crítica. La estandarización internacional nos lleva a la idea de la homogenización obviando y deslegitimando los contextos sociales diversos que caracterizan a los seres humanos.
} 
La deserción entendida como el abandono de los estudios y rezago escolar es producto de un conjunto de causas multidimensionales, las cuales se relacionan, entre otros con las condiciones económicas, académicas y metodológicos, pero también culturales e ideológicas, tal como lo expone Rodríguez (2007). De esta manera se ha concebido que la pobreza estructural es una de las principales causas que se asocian con diferentes problemáticas que afectan a un amplio sector de la población joven tales como: la deserción y la explotación comercial (trabajo infantil y comercio sexual) ${ }^{6}$.

Así, hoy en el sistema educativo formal, nos encontramos con la deserción escolar, expulsión o exclusión de muchos jóvenes que no vuelven a las aulas o que para finalizar la secundaria duran el doble de los años ${ }^{7}$. Varias son las razones vinculadas con los estilos de vida y condiciones económicas y culturales para que dicha situación se presente. El Informe del Estado de la Educación (2005), en Costa Rica, al respecto nos relata:

La juventud costarricense dedica más tiempo a ver televisión o a reunirse con amistades (más de cinco horas por semana en ambos casos para el $43 \%$ y $41,9 \%$ de los entrevistados) o incluso a visitar centros comerciales, que a la lectura (menos de dos horas por semana para el $50 \%$ ) o al estudio (menos de cinco horas semanales)... un $27,6 \%$ argumenta falta de interés en el aprendizaje formal (entre los hombres el porcentaje es de $31,6 \%$ ), un $16,3 \%$ indica que no puede pagar sus estudios, un $7,7 \%$ tiene que trabajar $(12,5 \%$ hombres $)$ o un $6,1 \%$ prefiere hacerlo $(8,5 \%$ de los hombres)...la mujeres reportan abandono de los estudios por embarazo o matrimonio $(8,4 \%)$, por problemas de acceso al sistema educativo (8\%) y por tener que ayudar en los oficios domésticos (6,9\%). (Programa Estado de la Nación, 2005, p. 71)

Continuando con el Informe reseñado el problema de la deserción se valora como una situación que persiste y lo tipifican como el abandono precoz del sistema educativo formal. Si bien el peso de las condiciones personales del estudiantado y su familias son factores que

\footnotetext{
${ }^{5}$ Punto que también requeriría un análisis crítico producto de la distribución desigual de recursos entre regiones.

${ }^{6}$ Córdoba (22 de junio 2006) en el artículo del Semanario Universidad plantea que "...se constata que todos los adolescentes entrevistados se encuentran en situación de explotación y no cuentan con las garantías sociales ni la supervisión requerida para el pago de salarios, jornada laboral y riesgos de trabajo. " (p.6, 22 al 28 de junio)

${ }^{7}$ Villegas (2 de junio 2006) presenta como síntesis que solo un $20 \%$ se gradúa sin repetir. El promedio que tarda un joven para graduarse es de 9,4 años. Asimismo, Villegas (28 de abril 2007) anota que la mayor deserción se registra en las instituciones de educación. Y el mismo periodista Villegas (4 de noviembre del 2006) hace un reportaje explicando que la salida se produce justamente en sétimo año.
} 
en la literatura se han considerado como importantes según podemos retomar en la siguiente cita, también, desde mi punto de vista, las instituciones educativas tienen un papel fundamental para prevenir y retener a los escolares, aspecto que parece que no se sabe muy bien cómo hacerlo.

EI MEP ha analizado los factores que inciden en el abandono escolar y entre estos ha identificado aquellos propios del estudiante, los de índole familiar-social y también los de carácter económico. Esta tipología coincide con el Informe Estado de la Nación a partir de la Encuesta de Hogares del INEC, cuyos resultados indican que la inasistencia a secundaria se explica no solo por la situación socio-económica sino también por la falta de interés en el sistema educativo formal. (Programa Estado de la Nación, 2005, p. 26)

Por esta razón, no es extraño que como promesas de campaña política el gobierno de Oscar Arias se haya planteado al inicio de su gestión que se implementaría el programa Avancemos $^{8}$. Programa que entrega recursos económicos a los jóvenes y sus familias con el compromiso de permanecer en el sistema educativo formal. Es interesante que Siu (5 de marzo 2007) al darle seguimiento al proyecto mencionaba que solo un $3 \%$ de del estudiantado había recibido el subsidio, quedando un porcentaje muy alto sin atención. Este es un programa que parece ofrecer respuestas a las necesidades económicas, y aún no se sabe si resolverá la aspiración a la universalización de la educación secundaria. Podemos mencionar que tal como se propone este es un problema multicausal que tienen otras aristas que hay que asumir.

Por otro lado, encontramos referencias a estudios que plantean que las personas que han desertado no tienen interés en el sistema educativo formal y lo refieren a lo poco atractivas que son la metodología o didáctica de aula. Siu (10 de agosto, 2006) al referir un estudio de la Universidad Nacional: "Actualmente los problemas económicos no serían la principal causa por la que los jóvenes dejan las aulas de secundaria, según concluye un estudio del Centro de Investigación en Docencia de la Universidad Nacional” (Siu, 2006, p. 10).

\footnotetext{
${ }^{8}$ Venegas (9 de mayo, 2006) en la cobertura que da al primer acuerdo del Poder Ejecutivo en el Periódico La Nación del mes de mayo.
} 
Asimismo, en un estudio en proceso de Kaufmann y Jaime (2008), encuentran que las personas que han desertado del sistema tienen un malestar producto de las relaciones interpersonales que encuentran en la secundaria en el marco de que si se ausentan o se van a nadie parece importarles. Y ellas concluyen: "Los entrevistados nos recuerdan que el proceso educativo no se reduce a la transmisión de conocimientos y destrezas, por el contrario, 'la educación es una tarea de sujetos, de relaciones, de intercambios, de lugares compartidos" (Kaufmann y Jaime, 2008, p 10).

Una política contra la deserción escolar y una gestión académico-administrativo tendría que considerar a las instituciones escolares de manera articulada e integrada con los diferentes actores como son las familias y el alumnado, los docentes, así como las propuestas de gestión en el marco de una propuesta colectiva donde la preocupación por el otro es un factor fundamental para apoyar las necesidades de las personas involucradas.

Esto nos lleva a preguntarnos, cuáles serán las prácticas y acciones que realizan los centros educativos que cuentan con bajos índices de deserción ${ }^{9}$. En el artículo de La Nación del mes de junio del 2007, de Villegas, Iglesias y Ángulo, nos refieren la existencia de cantones relativamente exitosos que cuentan con bajos índices de deserción: Flores ${ }^{10}$, Moravia, Santo Domingo, Alfaro Ruiz y Hojancha. En este artículo, al ser consultado el Ministerio de Educación Pública se recabó la siguiente información: "Alejandrina Mata, viceministra de Educación, dice que el factor que más provoca estas diferencias es el relacionado con el manejo del centro educativo" (Villegas, Iglesias y Ángulo, 2007, p 10 A).

Siguiendo está lógica de pensamiento surge la presente indagación a partir de la comprensión que la institución escolar como un sistema integrado en la comunidad y respondiendo a una política nacional tendría que contar con un proceso de gestión donde existan planes organizados para responder al artículo 57 del Código de la Niñez y la adolescencia que dice:

\footnotetext{
${ }^{9}$ Tema que sería interesante revisar para aprender de las buenas prácticas que se realizan y que no es tema de este trabajo.

10 “...en la escuela Ramón Barrantes, en Flores, pues si un alumno falta tres días consecutivos los docentes lo buscan o piden la intervención del Patronato Nacional de la Infancia (PANI) dijo la directora Alejandra Gutiérrez" (Villegas, Iglesias y Ángulo, 11 de junio, 2007)
} 
Permanencia en el sistema educativo El Ministerio de Educación Pública deberá garantizar la permanencia de las personas menores de edad en el sistema educativo y brindarles el apoyo necesario para conseguirlo.

Tarea que responde a una situación complicada y tal como lo hemos mencionado la deserción tiene que entenderse de manera integral, donde el problema incluye al sistema educativo como un todo y los diferentes entes que están interrelacionados como son las familias, el alumnado, la comunidad y el centro educativo (Abarca y Sánchez, 2005 y León, 2002 entre otros).

Así Rojas (2000, p. 2) propone que "la transformación en las instituciones educativas debe tener dos metas...aumentar la capacidad de la organización para adaptarse a los cambios del entorno y cambiar las conductas de las personas y grupos de la institución“. Es justamente a esta organización del centro educativo que se dirigió este trabajo, en el marco de una propuesta de los Derechos Humanos.

El estudio de León (2002) en una institución escolar muestra que los controles e información por parte de los docentes sobre la presencia o ausencia del estudiantado es prácticamente nula. Comenta la autora que los padres de familia asisten a la institución por los problemas de absentismo sólo cuando son convocados y no hay coordinación entre guías, orientadores y auxiliares administrativos. Los orientadores trabajan el problema de absentismo individualmente y si por razones de recargo de funciones no pueden llevar el seguimiento, dejan el caso. Parece ser que las instituciones escolares no logran coordinar sus acciones producto de un énfasis en los aspectos técnicos instrumentales que se sintetiza en la siguiente cita:

El problema es que los espacios para pensar la escolaridad están en función de los programas y los reglamentos, no necesariamente de la expresión del malestar. Faltar, por ejemplo, tiene una respuesta reglamentaria concreta, así que podemos suponer que las respuestas a veces ocurren cuando ya el alumnado ha dejado las aulas definitivamente. (Kaufmann y Jaime, 2008, p. 9)

Por otra parte, en el informe de la Escuela de Psicología de la Universidad de Costa Rica UCR - en conjunto con la Organización Internacional del Trabajo (OIT), del año 2005, sobre 
el proyecto para contribuir a la prevención y eliminación de la explotación sexual comercial en la zona sur, se plantea la importancia que tienen las instituciones escolares para la protección de las personas menores de edad: “... la permanencia en el sistema educativo se convierte en factor protector en la niñez y la adolescencia, a la vez que impulsa el desarrollo de habilidades y destrezas que promueven una mejor calidad de vida" (ESP/UCR y OITIPEC, 2005, p. 37).

Así, el producto de este trabajo giró alrededor de la opinión de un grupo de docentes ${ }^{11}$ a quienes se les preguntó sobre las actividades y acuerdos que existen institucionalmente para prevenir la deserción escolar. No se pretende con la información recabada generalizar la información a todo el sistema educativo. No obstante, en el marco de la exploración, se pueden dar pistas de lo que pueda estar ocurriendo en las escuelas. Así surgen más bien cuestionamientos que tendrían que ser abordados en otros estudios. $Y$ que podría contribuirse a realizar propuestas concretas.

En este trabajo se hizo un sondeo con el personal docente de seis instituciones escolares públicas (5 escuelas primarias y un colegio) en el marco de las actividades de un proyecto de Acción Social ${ }^{12}$ desarrollado desde la Universidad de Costa Rica en coordinación con el proyecto Primero Aprendo de la Pastoral Social-Cáritas.

La investigación nos acercó a las instituciones escolares, pensadas como un sistema organizado, donde se esperaba que las y los docentes contaran con políticas y procedimiento claros para prevenir la salida de la institución escolar en conjunto con otras instituciones nacionales que tienen como mandato proteger y hacer valer los derechos de la niñez y la adolescencia, tales como el Patronato Nacional de la Infancia (PANI). Los resultados nos brindan algunos puntos de reflexión, que más bien cuestionan la puesta en práctica del artículo 57 del Código de la Niñez y la Adolescencia ya mencionado y coincide con algunos de los estudios reportados.

\footnotetext{
${ }^{11}$ En este estudio, el personal docente y administrativo de las instituciones son la fuente principal de información para conocer la opinión que tienen sobre las acciones y acuerdos que como escuela o colegio tienen sobre la deserción escolar.

12 Cordero (2008) Proyecto ED-2036 denominado "Apoyo al proyecto Primero Aprendo. Una experiencia con docentes" el cual finalizó en el mes de marzo 2008.
} 
El trabajo sistematiza la opinión del personal docente de las instituciones visitadas y quizá allí radica la novedad, ya que la mayoría de las investigaciones que se han realizado retoman como énfasis y objeto de estudio a la población estudiantil afectada. Tal como se presenta en los resultados, las escuelas primarias tienen mayores recursos para mantener al alumnado a diferencia del único colegio visitado.

Sin embargo, Hernández (1999) explica desde un estudio en la zona Atlántica, que las escuelas no logran incorporar respuestas oportunas a las necesidades de las poblaciones que atienden. Por ejemplo, en el campo de las migraciones internas, el sistema educativo solo tiene disposiciones formales para la realización de los traslados y no se hacen planes de acción para atender a esta población. Entre otras conclusiones, plantea que la escuela como sistema se limita a responder a las demandas de matrícula y no realiza diagnósticos sobre la realidad de la comunidad, a pesar de que hay una ley que plantea que la educación primaria es obligatoria por ley. La asistencia a la escuela queda en el plano familiar y se ampara a la práctica de no intervenir en las familias.

De igual manera, Primero Aprendo y PREAL (2006) coinciden con los estudios referidos anteriormente, en las múltiples causas de la deserción, relacionados con la extraedad, las condiciones familiares y personales del estudiantado. Pero a la vez lo relacionan con las acciones del personal docente que no cuenta con el tiempo, la motivación, ni los recursos para mantener a los alumnos que trabajan en sus centros educativos.

\section{Metodología}

En el marco del proyecto de Acción Social ED-2036 denominado "Apoyo al programa "Primero Aprendo", una experiencia con docentes“ inscrito en el Vicerrectoría de Acción Social de la Universidad de Costa Rica (UCR), en coordinación con Pastoral Social Cáritas de Costa Rica y el Instituto de Investigación en Educación (INIE) de la UCR; durante el año 2007 se llevaron a cabo taller participativos, con grupos de docentes y administrativos de cinco escuelas y un colegio, con el objetivo de impulsar la discusión y la visión de la Educación en el marco de los Derechos Humanos y la incorporación de una metodología lúdico creativa a partir de la expresión artística al currículo escolar. 
El tipo de estudio es exploratorio y con un enfoque cualitativo, que utiliza la triangulación de la información para corroborar algunas ideas de cómo se están trabajando en el centro educativo la problemática que nos ocupa. Por tal razón, no se busca muestras estadísticamente significativas, sino valorar las opiniones de las personas como una forma de explicar lo que está aconteciendo en su trabajo cotidiano, bajo el supuesto que la escuela o colegio actúa como una organización integrada.

Así, el esfuerzo de investigación, se concibió como una oportunidad para realizar un sondeo de opinión, por medio de una encuesta dirigida al personal que asistió a los talleres ${ }^{13}$, estrategia que permitió conocer lo que piensan del problema y recabar algunas acciones que realizan para enfrentar la situación de la deserción escolar en sus lugares de trabajo.

Se logra socializar los resultados de la encuesta de opinión y generar una discusión posterior que se hizo en cada una de las instituciones escolares visitadas. Con base en un resumen de las principales conclusiones y utilizando una guía de preguntas, a manera de síntesis generadora, se recaban algunas ideas para complementar la información, ver anexo 1. Se administró la encuesta, como un medio para conocer la opinión de las personas que participaron y no se busca ser generalizable. Ahora bien se considera que desde el marco de investigación cualitativo si se logra exponer algunos indicios sobre lo que está ocurriendo en los centros educativos. El manejo de la información se dirige a presentar los datos más relevantes y se presentan los hallazgos más significativos que dan pie a una serie de conclusiones sujetas a discusión.

\section{Procedimiento}

En un primer momento, se administró la encuesta ${ }^{14}$ a la población que participó en el proyecto ED-2036, ya reseñado. La encuesta se completa en la primera actividad que dio inicio a los talleres programados en cada institución educativa. La discusión de los resultados de la encuesta se hizo en cada escuela o colegio y se basó en la síntesis que se presenta en el anexo 1. Dicha reflexión grupal se realiza en el último taller de cierre del proyecto mencionado. La autora del presente artículo estuvo en todas las etapas del proceso. Todas

\footnotetext{
${ }^{13}$ Las instituciones escolares participantes fueron las que el Programa Primero Aprendo de Pastoral SocialCáritas de Costa Rica venía trabajando. Por tal razón, no hubo una escogencia intencional de ellas, sino que se aprovecha la vinculación y se hace la indagación.
} 
las actividades fueron realizadas en las instituciones escolares cercanas al lugar de trabajo. Las personas mostraron interés por colaborar y en las discusiones grupales, los comentarios se relacionaron con ejemplos de la propia realidad cotidiana.

\section{Instrumentos.}

El enfoque metodológico se basó en un acercamiento exploratorio que utilizó como técnicas de trabajo la encuesta y el grupo de discusión a partir de una perspectiva de investigación cualitativa. No se pretende con la encuesta llegar a generalizaciones sobre la población de educadores del país. Sin embargo las opiniones de las personas que completan la información sirven para dar una idea de lo que está ocurriendo en cada institución educativa participante. Desde este marco y retomando algunas fuentes bibliográficas ${ }^{15}$ que apuntan a preguntar sobre el funcionamiento de los centros educativos y en relación con otras instituciones públicas, fue el interés conocer cómo se enfrenta la deserción escolar tanto a nivel del centro educativo como de los docentes.

\subsection{Encuesta:}

La encuesta contempló datos generales de las personas, así como se buscó responder a las siguientes preguntas:

- ¿Qué entienden por deserción escolar?

- ¿Qué acciones establecen como escuela y en su actividad cotidiana para enfrentar el problema?

- ¿Cuáles son las causas que determinan el abandono de la institución?

El material se codificó y procesó en el paquete SPSS con licencia del INIE. Una vez sistematizada la información se elabora una síntesis y se utiliza para la siguiente etapa. En el anexo 2 se encuentra la encuesta administrada.

\subsection{Grupos de discusión.}

La discusión grupal tomó como base los resultados de la encuesta, material que se incluye en el anexo 1. Dicha consulta buscó corroborar algunos datos y recabar más información sobre el tema, así como, comprender cómo se incorporan las políticas institucionales para

\footnotetext{
${ }^{14}$ Ver en anexo \#2 el cuestionario.

15 Primero Aprendo y PREAL, (2006), León (2002), Hernández (1999).
} 
enfrentar la deserción. Se presentaron tres postulados y cuatro preguntas como guía para la reflexión grupal.

En cinco centros educativos, se logra trabajar en subgrupos y en uno la reflexión grupal se realiza con el total de las personas asistentes. Se recabó la información por escrito y luego se sintetizó.

\section{Población.}

Se trabajó con docentes y personal administrativo de cinco escuelas y un colegio ubicadas en tres provincias del país. Dos escuelas primarias en la provincia de Guanacaste, dos en la provincia de Puntarenas, una escuela primaria y un grupo de docentes de un colegio técnico profesional de la provincia de San José. Cada una de las escuelas y el colegio están ubicadas en zonas marginales y rurales $^{16}$. En el cuadro \#1 se encuentran algunas características de las instituciones y la cantidad de personas que se les administró la encuesta.

\section{Resultados}

Los resultados se presentan con base a los datos de las encuestas señalando en algunas ocasiones las diferencias entre instituciones y en otros presentando las opiniones totales. En la segunda parte los resultados corresponden a la discusión grupal según los postulados y preguntas realizadas.

\section{Resultados de la encuesta.}

\subsection{Datos generales ${ }^{17}$.}

El grupo de docentes y administrativos que completaron las encuestas están referidos en el cuadro \#1, tal como se puede observar existió una mayor participación del personal docente y administrativo de las instituciones ubicadas en las provincias de Puntarenas con un $70 \%$ a $68 \%$ del total de cada institución escolar. En Guanacaste hubo una representación de un

\footnotetext{
${ }^{16}$ En el informe de Gish, Paz \& Asociados (2007) se encuentran las características del lugar y las condiciones de las Escuelas de Puntarenas. Y en el informe de Gish, Paz \& Asociados (2007) se encuentran las condiciones de la escuela ubicada en la zona fronteriza. Ambos trabajos son monografías que se encuentran en la siguiente dirección http://www.primeroaprendo.org/documentos?idclasificacion=4

17 Se opta por no mencionar las instituciones educativas, sin embargo en las referencias bibliográficas del Programa Primero Aprendo se puede revisar una mejor caracterización de tres de los seis centros educativos.
} Volumen 8, Número 3, Año 2008, ISSN 1409-4703 
$54 \%$ al $57 \%$ del personal. En la provincia de San José la participación fue menor en la escuela con un $22 \%$ y en el colegio llegó a un $42 \%$.

\section{Cuadro \#1}

Número de personas participantes y algunas características de las instituciones. Año 2007.

\begin{tabular}{|c|c|c|c|}
\hline $\begin{array}{l}\text { Instituciones } \\
\text { escolares }\end{array}$ & $\begin{array}{l}\text { Cantidad y } \\
\text { porcentaje de } \\
\text { participantes. }\end{array}$ & $\begin{array}{l}\text { Cantidad de personal de } \\
\text { la institución escolar. }\end{array}$ & 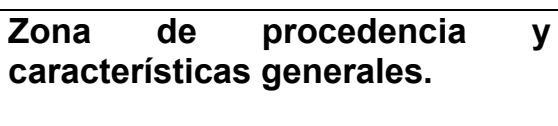 \\
\hline $\begin{array}{l}\text { Escuela \#1 } \\
\text { Puntarenas } \\
\text { (J) }\end{array}$ & $68,75 \%$ & $\begin{array}{l}48 \text { docentes y } \\
\text { administrativos. Cuenta con } \\
\text { equipo interdisciplinario. }\end{array}$ & $\begin{array}{l}\text { Urbana marginal en la ciudad de } \\
\text { Puntarenas. }\end{array}$ \\
\hline $\begin{array}{l}\text { Escuela \#2 } \\
\text { Puntarenas } \\
\text { (M) }\end{array}$ & $70,83 \%$ & $\begin{array}{l}24 \quad \text { docentes } \quad y \\
\text { administrativos. }\end{array}$ & $\begin{array}{l}\text { Urbana marginal en la ciudad de } \\
\text { Puntarenas. Ambas escuelas } \\
\text { están cercanas una de la otra. }\end{array}$ \\
\hline $\begin{array}{l}\text { Guanacaste } \\
\# 1 \text { (SC) }\end{array}$ & $\begin{array}{c}24 \\
54,54 \%\end{array}$ & $\begin{array}{l}41 \text { docentes } \\
\text { administrativos. }\end{array}$ & $\begin{array}{l}\text { Escuela en zona fronteriza con } \\
\text { Nicaragua. Extracción rural ligada } \\
\text { a la explotación del mono cultivo. }\end{array}$ \\
\hline $\begin{array}{l}\text { Guanacaste } \\
\text { \#2 (CJ) }\end{array}$ & $\begin{array}{c}44 \\
57,14 \%\end{array}$ & $\begin{array}{llr}70 & \text { personas } & \text { entre } \\
\text { docentes y administrativos. }\end{array}$ & $\begin{array}{l}\text { Escuela ubicada en Liberia en } \\
\text { zona marginal. }\end{array}$ \\
\hline $\begin{array}{l}\text { Escuela en } \\
\text { San José }\end{array}$ & $\begin{array}{c}16 \\
22,8 \%\end{array}$ & $\begin{array}{l}70 \quad \text { docentes } \\
\text { administrativos. }\end{array}$ & $\begin{array}{l}\text { Escuela ubicada en una zona } \\
\text { urbano marginal. }\end{array}$ \\
\hline $\begin{array}{ll}\text { Colegio en } \\
\text { San José }\end{array}$ & $\begin{array}{c}42 \\
42 \%\end{array}$ & $\begin{array}{l}100 \quad \text { docentes } \\
\text { administrativos }\end{array}$ & $\begin{array}{l}\text { Colegio Técnico Profesional en } \\
\text { zona rural de San José. }\end{array}$ \\
\hline
\end{tabular}

La frecuencia de respuestas según sexo corresponde a un $83 \%$ de mujeres y un $14.3 \%$ de varones. El lugar donde encontramos mayor cantidad de varones se presenta en el colegio con una presencia de un $26.2 \%$. En cuanto al grado académico reportado la mayoría de las personas son licenciadas y bachilleres, solo hay un grupo pequeño que cuenta con maestría (4.4\%). Hubo un $13,3 \%$ que no reportó su grado académico. Las personas con grado académico de solo primaria o secundaria, corresponde a $2,2 \%$ cuatro de ellas que posiblemente se desempeñan como guardas y conserjes.

Con respecto a la edad, encontramos que un 69,1 \% están ubicados entre 25 a 44 años y un $25 \%$ son mayores de 45 años, existiendo un pequeño grupo menor a 25 años con tan solo el $4.9 \%$. Esto nos lleva a pensar que son personas que cuentan con amplia experiencia en el campo educativo. 


\subsection{Conocimiento sobre el tema de la deserción.}

Un $87,4 \%$ de las respuestas mencionaron que si conoce casos de deserción. En cuanto a cómo la definen, los participantes comentan que ésta se entiende como dejar de asistir al centro educativo, ya sea porque abandona temporalmente los estudios $(78,7 \%)$. Tan solo un pequeño porcentaje $7,7 \%$, mencionó el trabajo infantil como una forma de sustitución del estudio, correspondiente a 14 personas. Por otra parte, hubo 10 respuestas, con un 5.4\% que consideraron que la deserción se debe a la desmotivación para asistir a la escuela e indiferencia hacia los estudios.

Con respecto a la pregunta a qué edad se debe terminar la primaria, el consenso es que debe ser entre los 12 a 13 años. Recordemos que algunos de los niños, niñas y adolescentes que están en las aulas abiertas donde funciona el programa Primero Aprendo ${ }^{18}$ cuentan con extra edad y son personas que tienen que realizan trabajo infantil.

En cuanto a la opinión sobre si las familias (madres, padres) son conscientes de los efectos de la deserción, solo un 40,7\% del personal docente piensa que sí, y un $44,5 \%$ que considera que no. La opinión sobre la existencia de una clara consciencia sobre las consecuencias de la deserción escolar por parte de las familias, sería un tema que debería ser retomado en futuros estudios, con el objetivo de comprender por qué las madres, padres o familiares cercanos no hacen nada para evitar esta situación. Percepción que por supuesto no necesariamente es coincidente con lo que podrían estar contemplando los familiares de la persona que haya desertado del sistema educativo.

La opinión sobre la percepción del problema de la deserción escolar varía según sea el ámbito de acción. Así encontramos que existe una percepción diferente si corresponde al centro educativo o si responde a lo que se hace en el aula. En el cuadro \#2 se presentan las diferencias de opinión. El problema de la deserción en el aula es considerado como "poco (52\%) o nada (14,83\%)". Cuando la pregunta sobre la deserción se ubica en la institución educativa, la percepción es mayor colocando como mucho un $46 \%$. No sabemos si esto responde a la idea de que ser mal maestro o mala maestra, podría significar tener un porcentaje algo de deserción en su aula escolar. 


\section{Cuadro \#2}

Percepción de la magnitud de la deserción según centro educativo o la propia aula. Año 2007

\begin{tabular}{|l|c|c|}
\hline Respuestas & $\begin{array}{l}\text { Percepción de la magnitud del } \\
\text { problema de deserción en la } \\
\text { institución escolar }\end{array}$ & $\begin{array}{l}\text { Percepción de la magnitud del } \\
\text { problema de la deserción en el } \\
\text { aula escolar }\end{array}$ \\
\hline Mucho & $46 \%$. & $23,07 \%$ \\
\hline Poco & 47,6 & 52,2 \\
\hline Nada & 1 & 14,83 \\
\hline No respuesta & 5,4 & 9,9 \\
\hline Total & 100 & 100 \\
\hline
\end{tabular}

No obstante, los datos anteriores en Puntarenas los participantes consideran que hay pocos casos de deserción escolar. En la escuela \#2 en Guanacaste y la escuela en San José consideran que si hay mucho problema de este tipo. Esto nos lleva a cuestionar cómo trabajar estos temas cuando se considera que no pasa en el aula o en la escuela.

Con respecto a la pregunta: ¿qué hace la institución escolar, donde usted trabaja para evitar la deserción escolar?, las respuestas recaen en el trabajo de los equipos interdisciplinarios, con un $31,3 \%$ del total de respuestas. Seguido por las visitas al hogar con un $25,3 \%$, ayudar a las familias tan solo se consideró un $8,8 \%$. Es interesante que solo en cinco respuestas se mencionara la denuncia como una medida para evitar la deserción escolar.

Esta misma pregunta pero ahora ubicada en el aula y presentada en el cuadro \#3, las respuestas van en dos sentidos por una parte mejorar el trabajo didáctico de la clase, con un énfasis y control de parte de las y los docentes $(48,9 \%)$ cuya idea principal esta vinculada con la motivación de: el aprendizaje, la asistencia y el estudio; así como contar con un ambiente acogedor en el aula y el uso de técnicas innovadoras. Por otra parte, comunicarse con las madres y los padres, así como visitas al hogar se presentan con un $26,37 \%$. Este último dato es muy importante pues aún las maestras realizan la visita al hogar como una tarea propia, mientras que en la secundaria esto suele ser una tarea del departamento de orientación o del equipo interdisciplinario y no así de los docentes.

\footnotetext{
${ }^{18}$ Proyecto que está a cargo de la Pastoral Social Cáritas con quienes coordinamos para realizar la actividad desde la INIE. Primero Aprendo y PREAL (2006)
} 


\section{Cuadro \# 3}

¿Qué hace usted en el aula para evitar la deserción escolar? Año 2007

\begin{tabular}{|l|c|c|}
\hline \multicolumn{1}{|c|}{ Respuestas } & Frecuencia & Porcentaje \\
\hline Motivar la asistencia & 17 & 9,34 \\
\hline Brindar un ambiente acogedor & 9 & 4,95 \\
\hline Motivar el aprendizaje & & 19,23 \\
\hline Dar a conocer la importancia de los estudios & 5 & 15,38 \\
\hline Concientizar y comunicarse con los padres & 28 & 20,33 \\
\hline Utilizar técnicas motivadoras & 37 & 5,49 \\
\hline Visitas al hogar & 10 & 6,04 \\
\hline Otro & 11 & 6,04 \\
\hline Nada & 11 & 2,75 \\
\hline Total & 5 & 89,56 \\
\hline No respuesta & 163 & 10,44 \\
\hline Total & 19 & 100,00 \\
\hline
\end{tabular}

\subsection{Causas de la deserción.}

Se les solicitó en la encuesta a las y los docentes que anotarán las principales causas de la deserción escolar, encontramos que hay una proporción de respuestas que mencionan a los problemas económicos de las familias como una limitante, con más de un $50 \%$. El peso, por lo tanto, está en las familias y sus condiciones de vida. Aspecto en el que no estuvo de acuerdo el grupo de participantes de la escuela ubicada en San José pues comentaron que en el barrio hay muchas ayudas de parte de instituciones públicas así como privadas y que la razón es el desinterés de las familias. Situación que no se encontró en los otros centros educativos.

No obstante en su conjunto las principales causas mencionadas de la deserción escolar de acuerdo al orden de importancia reportado son las siguientes:

1. Falta de recursos económicos en el seno familiar para mantener los gastos en materiales necesarios. (Carga económica para la familia)

2. Reemplazan la escuela por el trabajo.

3. Labores en el hogar impiden un desarrollo adecuado en las labores de la Escuela.

4. Repiten el grado.

5. Motivación y participación de los padres en el proceso educativo. 
6. Carencia de materiales didácticos adecuados.

7. Metodología de trabajo en el aula.

8. Contenidos de los programas educativos.

9. Mal estado de la infraestructura escolar.

Con respecto a la pregunta sobre cuáles son las principales dificultades que se encuentra para evitar la deserción escolar, la respuesta recae en el desinterés familiar, seguido de los problemas de orden económico, dejando el problema fuera del centro educativo. Esto parece problemático pues podría traer sentimientos de impotencia hacia la situación. En el cuadro \#4 se encuentra el detalle de esta respuesta que es diferente a la anterior pero que está relacionada.

\section{Cuadro \# 4}

\section{Principales obstáculos planteados para evitar la deserción.}

Año 2007

\begin{tabular}{|l|c|c|}
\hline \multicolumn{1}{|c|}{ Respuestas } & Frecuencia & Porcentaje \\
\hline Comunicación con los padres & 14 & 7,69 \\
\hline Migración familiar & 6 & 3,30 \\
\hline Trabajo infantil & 3 & 1,65 \\
\hline Falta de actividades y talleres sobre el tema & 2 & 1,10 \\
\hline Falta de apoyo e interés familiar & 58 & 31,87 \\
\hline Desintegración familiar & 2 & 1,10 \\
\hline Sobrecarga de trabajo a los docentes & 4 & 2,20 \\
\hline La situación económica & 39 & 21,43 \\
\hline Problemas de aprendizaje en los y las estudiantes & 1 & 0,55 \\
\hline Falta de compromiso de las instituciones y docentes & 3 & 1,65 \\
\hline Ignorancia & 4 & 2,20 \\
\hline Falta de material didáctico y de programas educativos & 1 & 0,55 \\
\hline Ambiente que rodea el estudiante & 2 & 1,10 \\
\hline Zona de marginación & 1 & 0,55 \\
\hline Otro & 10 & 5,49 \\
\hline Total & 150 & 82,42 \\
\hline No respuesta & 32 & 17,58 \\
\hline Total & 182 & 100,00 \\
\hline
\end{tabular}

¿Qué aspectos de nuestra práctica educativa pueden significar un obstáculo para que niñas, niños y adolescentes sientan la necesidad de abandonar la escuela? En orden de respuesta 
el grupo en su totalidad mencionó la relación docente, seguida de la relación con las y los compañeros, quedando como tercera opción el material didáctico y metodología en el aula. Esto nos indica que hay conciencia de la importancia de las relaciones interpersonales y del papel de la maestra y el maestro. Relacionado con este punto, en la discusión grupal que se hizo posterior surgió la preocupación de cómo establecer las relaciones con las familias, ya que existe el temor, por ejemplo, en las zonas de Puntarenas, sobre la protección que pueden tener las y los docentes a la hora de hacer las visitas al hogar. Aspecto que no se encuentra en la escuela de San José, pues parece que se sienten más protegidas por instituciones como el Patronato Nacional de la Infancia y la oficina local del lugar.

\subsection{Conclusiones generales del cuestionario.}

El tema de la deserción parece no estar articulado a una política escolar, que incluya un claro procedimiento. El foco de problema recae en las condiciones de vida de las familias o el desinterés de las familias. Esta fue una pregunta que se investigó en la siguiente sesión con los grupos participantes y que se detalla posteriormente.

Es interesante que un $84,1 \%$ consideren la deserción como una violación al derecho a la educación, tal cual podemos confirmar en el Código de la Niñez y la Adolescencia. Las y los docentes manifestaron que si promueven el derecho a la Educación. No obstante, pareciera que el énfasis de trabajo está en acciones centradas en el aula, y para una tercera parte de las encuestas, la tarea le corresponde a los equipos interdisciplinarios.

Esto nos llevó a preguntarles sobre la existencia de algún protocolo que pueda conocer la maestra o maestro, profesora o profesor, cuando se presentan casos de deserción.

\section{Resultados del trabajo Grupal.}

\subsection{Metodología del trabajo.}

Después de pasar las encuestas y sistematizarlas, se vuelve con el material del anexo 1 para trabajar en pequeños grupos los tres postulados y la cuatro preguntas que sirvieron de guía. La presentación de la información parte de las respuestas a los postulados y preguntas planteadas. En esta etapa se logran comprender con mayor detalle las diferencias entre lo que ocurre en las escuelas y el colegio. 


\subsubsection{Postulado $1^{19}$}

El postulado primero se confirma, y se plantea que el problema de la deserción no está articulado a una política escolar. No se cuenta con un procedimiento o protocolo que indique, a las maestras y maestros ¿qué hacer cuando un niño sale de la escuela?

La mayoría expone que no existen procedimientos a seguir, no hay protocolos. Solamente la escuela ubicada en San José manifestó que si los tienen y que ellas como docentes se reconocen como las primeras responsables para buscar y traer al niño o la niña de regreso.

¿Qué se hace para evitar la deserción? Es un aspecto que es confuso en la institución secundaria. En el colegio, el profesorado se queja de la cantidad y sobre carga de trabajo que tienen, producto de impartir clase a muchos y numerosos grupos. Si bien reconocen que un paso para la deserción es el ausentismo, no tenían claro a quién le corresponde la tarea de darle seguimiento. Consideran que es una tarea administrativa que no les corresponde a ellos como docentes.

Para la secundaria las visitas al hogar no se convierten en una labor asumida por la persona del docente, más bien ésta suele estar concentrada en el departamento de orientación. Por otra parte, las orientadoras comentaron que debería existir una coordinación entre ellas y la profesora o el profesor guía de cada sección. Al menos en este caso fue evidente que no existen políticas ni acciones claramente definidas y por ello da la impresión que la problemática no es asumida por nadie.

En el caso de la escuela primaria las y los docentes tienen un desconocimiento de las leyes relacionadas con el tema y como inquietud principal surge la pregunta: ¿cómo nos podemos proteger legalmente cuando realizamos una visita domiciliaria por causa de agresión?

\subsubsection{Postulado $2^{20}$}

Con respecto a las causas de la deserción, surgió por una parte, a manifestar que es un asunto más bien relacionado con la negligencia de parte de las familias más que por asuntos

\footnotetext{
${ }^{19}$ El problema de la deserción no esta articulado a una política escolar. No se cuenta con un procedimiento o protocolo que nos indique, a las maestras y maestros ¿qué hacer cuando un niño sale de la escuela?

$20 \mathrm{El}$ problema principal recae en las condiciones de vida de las familias (generalmente por asuntos de económicos). Esta idea nos inhibe a realizar acciones fuera del aula para luchar contra la deserción, ya que pensamos que es inevitable.
} 
de orden recursos económicos. En la escuela ubicada en San José se enfatizó en este aspecto agregando que de manera particular en dicha localidad hay muchas ayudas económicas provenientes de instituciones públicas y privadas tanto nacionales como extranjeras.

Otro sector manifestó otra clase de dificultades como: el trabajo infantil, el desinterés de los estudiantes, el debilitamiento de un sistema de asistencia social: IMAS, PANI, FONABE. Podríamos mencionar que hay dificultades de parte de las instituciones para cumplir con su papel o que la lejanía de la capital produce que exista menos posibilidad de ayuda económica asistencial, para resolver las dificultades monetarias del estudiantado.

La idea sobre la carencia de recursos económicos para otro grupo sigue siendo una de las causas de la deserción. Así se mencionó, que la salida del niño o la niña es por un asunto de sobrevivencia familiar, si no sale a trabajar pueda que los miembros del grupo familiar se mueran de hambre.

\subsubsection{Postulado $3^{21}$}

Con respecto al tercer postulado hubo acuerdo en que la deserción escolar es violatorio de los Derechos Humanos. También expresaron que debe ser una tarea en conjunto: la escuela (docentes, equipos interdisciplinarios y dirección), las familias y las comunidades.

Por otra parte, se mencionó que muchos docentes no conocen los protocolos de denuncia a la fiscalía y solo una escuela manifestó la eficiencia de la oficinal del Patronato Nacional de la Infancia en acudir a responder a las denuncias que se ellas hacen.

\subsubsection{Pregunta $1^{22}$}

Con respecto a la pregunta de cuáles son las responsabilidades de las y los funcionarios del Ministerio de Educación Pública (MEP) para hacer efectivo del artículo 57 del Código de la

\footnotetext{
${ }^{21}$ Un $84,1 \%$ de las encuestadas considera que la deserción es una violación al derecho a la educación, tal cual se confirma en el Código de la Niñez y la Adolescencia. Las y los docentes manifiestan que si promueven el derecho a la Educación. Asimismo una mayoría comenta que las acciones principales para evitar la deserción le corresponden a los equipos interdisciplinarios.

22 ¿Cuáles son las responsabilidades que una funcionaria o funcionario del MEP tienen, para hacer efectivo el cumplimiento del artículo $57 ?$
} 
Niñez y la Adolescencia ${ }^{23}$. Las respuestas exponen la importancia de tener un control eficiente sobre las ausencias de las y los estudiantes. Además, están las acciones de visitar, motivar y velar porque el niño o la niña asistan a la clase. Sin embargo, esto no está tan claro en la secundaria estudiada, tal como se expuso anteriormente y de acuerdo al estudio de León (2002).

\subsubsection{Pregunta $2^{24}$}

En relación a ¿Cómo se integran la escuela, las familias y las instituciones para hacer efectivo el derecho a la educación? Responden que por medio de diversas actividades. Entre ellas se reconoce la importancia de hacer un trabajo sistemático con las familias y las personas de las comunidades; de mantener una permanente comunicación. No obstante, un grupo mencionó que el estudio del expediente no siempre funciona pues hay poca asistencia de parte de los padres de familia. Y en el colegio la queja de mucho trabajo prevaleció sobre una posibilidad de realizar los docentes algo más de su parte que el trabajo de aula.

\subsubsection{Pregunta $3^{25}$}

En el caso de la tercera pregunta: ¿Cuál tiene que ser el rol de la y el educador ante la deserción de un estudiante?, las respuestas fueron muy congruentes con el deber ser.

Se menciona que el docente por lo general sabe de la situación del o la estudiante y que esto lo obliga a tener un rol activo, de investigador. Además, si sabe del caso debe comunicarlo al equipo interdisciplinario. Hacer valer las leyes y lograr que se mantenga en la escuela. Para las profesoras y profesores, la administración del colegio debería darle el seguimiento al estudiantado e informarles de la situación de las y los alumnos.

\footnotetext{
${ }^{23}$ Artículo 57: Permanencia en el sistema educativo. El Ministerio de Educación Pública deberá garantizar la permanencia de las personas menores de edad en el sistema educativo y brindarles el apoyo necesario para conseguirlo.

${ }^{24}$ ¿Cómo se integran la escuela, las familias y las instituciones para hacer efectivo el derecho a la educación? Expliquen.

${ }^{25}$ ¿Cuál tiene que ser el rol de la y el educador ante la deserción de un estudiante?
} 


\subsubsection{Pregunta $4^{26}$}

En relación con las tares que desarrollan los equipos interdisciplinarios para enfrentar el problema de la deserción. Resulta que no en todas las instituciones educativas cuentan con este recurso humano. Sin embargo, en términos generales parece existir un desconocimiento de lo que hacen, quizá producto de una falta de comunicación.

En una de las escuelas, donde estuvo presente un profesional del equipo interdisciplinario respondió que las tareas de los equipos interdisciplinarios son en tres áreas: social, psicológica y orientativa. Esto los lleva a realizar visitas al hogar y en algunas situaciones tienen que hacer denuncias.

\section{Conclusiones y recomendaciones.}

Parece ser que en las escuelas que participaron en este estudio existe una mejor claridad sobre qué hacer cuando hay deserción. No obstante, ello no garantiza que todas las escuelas tengan como prioridad el prevenir la deserción.

En el caso del colegio visitado el tema de la deserción es confuso y existe poca reflexión sobre qué hacer. León (2002) en su estudio plantea problemas similares en relación con quién informa oportunamente de las ausencias del alumnado. Indica como lo plantea Hernández (1999) la idea que son las familias y el estudiante los responsables de asistir al centro educativo. Esta situación será una razón más de que la deserción en la educación secundaria es mayor que en primaria. Por ejemplo, las y los profesores relataban que hay alumnos y alumnas que suelen no asistir a sus clases pero que deambulan por el colegio y no parece suceder nada hasta que pierden el año. Asimismo, después de varios meses de ausencia se les informa que ya no están en la institución sin haber hecho de su parte nada al respecto. De manera honesta comentan que en algunos casos donde él o la alumna es de difícil manejo, cuando deserta hay un alivio por no contar más con su presencia. De igual manera las compañeras de orientación comentaron lo difícil que es obligar al alumnado a

${ }^{26}$ ¿Cuáles son las tareas que desarrollan los equipos interdisciplinarios para enfrentar el problema de la deserción? 
quedarse en el colegio cuando manifiestan que no les interesa las carreras técnicas que se imparten, en este centro educativo.

Lo que si resulta claro en el proceso es que no existe una política clara de qué hacer cuando deserta un estudiante unido a la ausencia de protocolos que pudieran mostrar todo el proceso (Primero Aprendo y PREAL, 2006). Tarea pendiente que requiere ser trabajada y liderada desde el Ministerio de Educación Pública, en el marco de la administración en la educación.

Entre otros aspectos que surgieron fue el temor de algunas personas a ser agredidas por las familias y la poca o nulo apoyo interinstitucional ${ }^{27}$. Esto hace que la deserción escolar se convierta en un camino más difícil de resolver cuando una profesional de la educación plantea que no realiza visitas al hogar por miedo a su integridad física.

Se requieren de acciones más orgánicas y una política institucional que contemple, al docente y al centro educativo en el conjunto con otros entes. Sería ideal realizar un esfuerzo por premiar aquellos lugares que se propongan una política de eliminación de las deserciones escolares.

El trabajo preventivo es fundamental y para ello se necesita establecer una cultura organizativa que pueda como conjunto ponerse de acuerdo de cómo enfrentar la deserción. Aunque no se puede concluir que el sistema educativo presenta las dificultades para la prevención y atención a esta problemática, si es válido señalarlo como un problema que requiere la gestión desde el centro educativo para responder a la propia legislación nacional.

Una conclusión clave en todo esto es que hemos avanzado mucho en la legislación sobre niñez y adolescencia pero aún no se sabe cómo se traduce en la práctica. En este caso el Ministerio de Educación Pública debería tomar acciones más articuladas para promover una cultura organizativa más propositiva.

${ }^{27}$ En la nota del periódico La Nación de Iglesia (11 de junio del 2007) se relata esta una situación donde el director manifiesta que fue amenazado por obligar a los padres a enviar a tres niños a la escuela. Volumen 8, Número 3, Año 2008, ISSN 1409-4703 
Coincidiendo con Salas (2006) en la presentación que realiza sobre la formación en administración educativa, menciona la importancia de los recursos del personal docente y del estudiantado, pero también indica que otro aspecto fundamental es el medio en que está inserto.

...Ésta, al igual que los docentes y estudiantes, debe formar parte de las prioridades de quien administra la educación para que tenga la sensibilidad y el conocimiento suficiente de su entorno y procure reducir las desventajas socioculturales que impactan el rendimiento académico. (Salas, 2006, p. 67)

La tarea de bajar la deserción es compleja por ello se necesita establecer las coordinaciones interinstitucionales y el apoyo de organizaciones comunales para enfrentar la problemática. Cuando estas coordinaciones son efectivas las y los docentes se sienten con autoridad para defender el derecho a la educación. Cuando no es así hay una sensación de soledad e impotencia. Por ello debe verse a los centros educativos como redes de interacción que de manera organizada y bajo una micropolítica, como lo plantea teóricamente Terrén (2004) establece redes de comunicación que construyen una comunidad articulada. En este sentido se requiere entender la importancia de las redes de comunicación y el foco en las relaciones interpersonales que brindan romper con el anonimato que parece ser la tónica en la secundaria (León, 2002 y Kaufmann y Jaime, 2008) a diferencia de la escuela primaria, la cual aún mantiene las relaciones cara a cara como una fuente de prevención a la deserción. 


\section{Referencias}

Abarca Rodríguez, Allan y Sánchez Vindas, M. Alejandra. (2005, 30 de setiembre). La deserción estudiantil en la educación superior: el caso de la Universidad de Costa Rica. San José: Actualidades Investigativas en Educación, 5 (Especial). http://revista.inie.ucr.ac.cr/articulos/extra-cea/archivos/desercion.pdf

Cordero Cordero, Teresita. (2008). Informe de Acción Social. ED-2036 Apoyo al Proyecto Primero Aprendo. Una experiencia con docentes. San José, Costa Rica. INIE, Vicerrectoría de Acción Social. Universidad de Costa Rica.

Córdoba Morales, Javier. (22 al 28 de junio 2006). Trabajo infantil agrícola saca a varones de las aulas. San José: Semanario Universidad, p.6.

Escuela de Psicología UCR y Organización Internacional del Trabajo (IPEC). (2005). Sistematización de experiencia. Proyecto: contribución a la prevención y eliminación de la explotación sexual comercial de niñas, niños y adolescentes en las comunidades de Corredores y Golfito. San José: Universidad de Costa Rica y OIT-IPEC.

Espíndola, Ernesto y León, Arturo. (2002). La deserción escolar en América Latina: un tema prioritario para la agenda regional. OEl: Revista Iberoamericana de Educación, (30). http://www.rieoei.org/rie30a02.htm

Fonseca Calvo, María Eugenia y Blanco Picado, Patricia. (2008). Noticia sobre el Primer Informe sobre el Estado de la Libertad de Expresión en Costa Rica. San José: Oficina Observatorio de la Libertad de Expresión. Facultad de Derecho de la UCR. Recuperado el 9-09-08 de la página principal, sección noticas de la Universidad de Costa Rica. www.ucr.ac.cr

Garbanzo Vargas, Guiselle M., y Orozco Delgado, Víctor H. (2007). Desafíos del Sistema Educativo Costarricense: un nuevo paradigma de la administración de la educación. San José. Educación: Revista de la Universidad de Costa Rica, 31 (2), 95-110.

Gajardo, Marcela. (2005). América Latina: políticas educativas de acción afirmativa. Situación, tendencias, perspectivas. García-Huidobro, Eduardo. Reseña Seminario Internacional sobre Políticas Educativas y Equidad. Santiago, Chile: UNICEF. Gráfica Funny.

Gish, Paz \& Asociados. (2007). Informe final de validación aprendiendo desde la solidaridad. Santiago, Atitlán: Guatemala, Junio. Recuperado el 14 de setiembre 2008, de http://www.primeroaprendo.org/documentos?idclasificacion=4.

Gish, Paz \& Asociados. (2007). Informe final de validación aula abierta intercultural. Santiago, Atitlán: Guatemala, Junio. Recuperado el 14 de setiembre 2008, de http://www.primeroaprendo.org/documentos?idclasificacion=4

Hernández C. Omar. (1999). Concepciones, disposiciones y prácticas hacia el capital escolar en un poblado liniero del Caribe costarricense. San José, Costa Rica. Educación: Revista de la Universidad de Costa Rica, 23 (2), pp. 175-188. 
Iglesias, Laura. (2007, 11 de junio). Director amenazado por obligar a niños a ir a clases. San José: Periódico La Nación, p. 10 A.

Kaufmann Kappari, Etty y Jaime Artega, Milagros. (2008). Los nombres de la exclusión escolar. San José: Instituto de Investigación Social, Ponencia presentada en las V Jornadas de Investigación 2008 viernes 19 de septiembre.

León Sólis, María Alicia. (2002). Factores administrativos que inciden en el absentismo a lecciones, en los estudiantes del Liceo de Escazú. Tesis Maestría profesional con mención en Administración Educativa. Universidad de Costa Rica. Sistema de Estudios de Posgrado. Escuela de Administración Educativa. San José, Costa Rica.

Machado, Ana Luiza; Palacios, María Amelia; Crotti Egidio y García Huidobro, Juan Eduardo. (2005). Introducción. García-Huidobro, Eduardo. Reseña Seminario Internacional sobre Políticas Educativas y Equidad. Santiago, Chile: UNICEF. Gráfica Funny.

Rojas, María Eugenia. (2000, Junio-septiembre). La deserción escolar en Costa Rica: un estudio de causas y consecuencias en una institución educativa. San José: Diálogos Revista Electrónica de Historia, 1, número 004. Disponible en http://historia.fcs.ucr.ac.cr/articulos/desersion.htm.

Rodríguez Amor, Randall. (2007). La violencia simbólica y la deserción escolar: el caso de los décimos años del Liceo de Cot de Oreamuno, Cartago. Anteproyecto de tesis aprobado por la Escuela de Sociología de la Universidad de Costa Rica. San José, Costa Rica.

Salas, Madriz, Flora Eugenia. (2006). Los estudios de seguimiento de la población graduada como herramienta para el cambio y la innovación en el currículo universitario: La experiencia de la escuela de administración educativa de la Universidad de Costa Rica. San José. Educación: Revista de la Universidad de Costa Rica, 30 (2), 6381.

Primero Aprendo y PREAL. (2006). Opciones educativas para la niñez trabajadora. Costa Rica. Recuperado el 14 de setiembre 2008, de http://www.primeroaprendo.org/documentos?idclasificacion $=5$

Programa del Estado de la Nación. (2005). Estado de la Educación en Desarrollo Humano Sostenible. San José: Consejo Nacional de Rectores.

Siu, María. (2007, 5 de marzo). Programa Avancemos llegó a un 3\% de jóvenes. San José: Periódico La República, p 8.

Siu, María. (2006, 10 de agosto). Clases poco atractivas provocan la deserción. San José: Periódico La República, p 10.

Terrén, Eduardo. (2004). Las organizaciones educativas como sistemas de comunicación. Un enfoque micropolítico. Revista Iberoamericana de Educación. Editada por Número 36: Septiembre-Diciembre. 189-214. Recuperada el 15 de setiembre 2008 de http://www.rieoei.org/rie36.htm. 
Venegas, Ismael. (2006, 9 de mayo) Gobierno ayudará a madres para evitar la deserción colegial. San José. Periódico La Nación, p. 10 A.

Villegas, Jairo. (2007, 28 de abril) Uno de cada 14 alumnos deserta de las escuelas y colegios. San José: Periódico La Nación, p. 10 A.

Villegas, Jairo; Iglesias, Laura y Ángulo, Francisco. (2007, 11 de junio). Brecha educativa amplía desinterés de padres y maestros. San José: Periódico La Nación, p. 10 A.

Villegas, Jairo. (2006, 4 de noviembre) 27.000 jóvenes dejaron las aulas después de vacaciones. San José: Periódico La Nación, p. 5 A.

Villegas, Jairo. (2006, 2 de junio) 535 de jóvenes de 17 años están fuera de las aulas. San José: Periódico La Nación, p. 10 A. 


\section{Anexo 1. Material de discusión grupal.}

\section{Resultados de las encuestas sobre deserción escolar.}

La deserción fue definida como dejar de asistir al centro educativo, abandonar temporalmente los estudios.

Revisar los siguientes resultados de la encuesta sobre deserción escolar.

- Trabajar en grupo las conclusiones con las preguntas en la página \#2.

- Escribir las respuestas del grupo y presentarlo.
Información de 182 formularios.

Escuelas y Colegio participantes:

Escuela \#1 (J) Puntarenas.

Escuela \#2 (M), Puntarenas.

Escuela \#1 (SC), Guanacaste.

Escuela \#2 (CJ). Guanacaste.

1. Las principales causas mencionadas de la deserción escolar de acuerdo al orden de importancia reportado son:

Las primeras causas son de económicas que sufren las familias.

1. Falta de recursos económicos en el seno familiar para mantener los gastos en materiales necesarios. (carga económica para la familia)

2. Reemplazan la escuela por el trabajo.

3. Labores en el hogar impiden un desarrollo adecuado en las labores de la Escuela.

Causas de rendimiento escolar, trabajo en el aula y escuela.

4. Repiten el grado.

5. Motivación y participación de los padres en el proceso educativo.

6. Carencia de materiales didácticos adecuados.

7. Metodología de trabajo en el aula.

8. Contenidos de los programas educativos.

9. Mal estado de la infraestructura escolar.

2. Las causas que podrían afectar en el aula según orden de importancia:

1. La relación con la maestra o maestro.

2. La relación con las y los compañeros. 
3. La metodología en el aula.

4. Material didáctico.

3. Las respuestas a la pregunta ¿qué tan frecuente es la deserción y repetición en su escuela, en su aula? la mayoría respondió que es poca.

4. Las respuestas sobre ¿qué hace la institución escolar, donde usted trabaja para evitar la deserción? La tarea recae en el equipo interdisciplinario, con $31,3 \%$ de las respuestas. Las visitas al hogar le siguen con un $25,3 \%$. Solo 5 respuestas manifestaron que hacen la denuncia.

5. ¿Qué hace usted en el aula para evitar la deserción? Acciones relacionadas con el trabajo de aula con un 48,9\%. Y las visitas al hogar con un $26.3 \%$. 
Revisar los siguientes postulados y responder a las preguntas. Esto tiene como objetivo llegar conclusiones sobre el tema de la deserción escolar.
Tomando en cuenta el tema de deserción

\begin{tabular}{|l|l|}
\hline Postulado & $\begin{array}{l}\text { Estamos de acuerdo o no con el } \\
\text { postulado, favor explica por qué. }\end{array}$ \\
\hline 1. El problema de la deserción no está articulado a una \\
política escolar. No se cuenta con un procedimiento o \\
protocolo que nos indique, a las maestras y maestros \\
¿qué hacer cuando un niño sale de la escuela? \\
\hline $\begin{array}{l}\text { El problema principal recae en las condiciones de vida } \\
\text { de las familias (generalmente por asuntos de } \\
\text { económicos). Esta idea nos inhibe a realizar acciones } \\
\text { fuera del aula para luchar contra la deserción, ya que } \\
\text { pensamos que es inevitable. } \\
\text { 3. Un 84,1\% de las encuestadas considera que la } \\
\text { deserción es una violación al derecho a la educación, tal } \\
\text { cual se confirma en el Código de la Niñez y la } \\
\text { Adolescencia. } \\
\text { Las y los docentes manifiestan que si promueven el } \\
\text { derecho a la Educación. Asimismo una mayoría comenta } \\
\text { que las acciones principales para evitar la deserción le } \\
\text { corresponden a los equipos interdisciplinarios. }\end{array}$ \\
\end{tabular}

Ante el artículo 57 del Código de la Niñez y la adolescencia que dice:

Permanencia en el sistema educativo El Ministerio de Educación Pública deberá garantizar la permanencia de las personas menores de edad en el sistema educativo y brindarles el apoyo necesario para conseguirlo.

Responda las siguientes interrogantes para en relación a su institución educativa y su experiencia docente. 
1. ¿Cuáles son las responsabilidades que una funcionaria o funcionario del MEP tienen, para hacer efectivo el cumplimiento del artículo 57?

2. ¿Cómo se integran la escuela, las familias y las instituciones para hacer efectivo el derecho a la educación? Expliquen.

3. ¿Cuál tiene que ser el rol de la y el educador ante la deserción de un estudiante?

4. ¿Cuáles son las tareas que desarrollan los equipos interdisciplinarios para enfrentar el problema de la deserción?

MUCHAS GRACIAS POR SUS RESPUESTAS. 
Anexo 2. Encuesta.

Universidad de Costa Rica Instituto de Investigación en Educación INIE

\#

Estimadas maestras y maestros el siguiente cuestionario es anónimos y tiene el objetivo de conocer su percepción y conocimiento del problema de la deserción escolar y la promoción del derecho a la educación. Esto nos permitirá darle un seguimiento al trabajo sobre la Educación Inclusiva.

Datos generales.

\begin{tabular}{|c|c|c|c|}
\hline \multicolumn{4}{|l|}{20} \\
\hline Categorias & \multicolumn{2}{|r|}{ Respuestas } & Cód. \\
\hline 1. Grado académico & \multicolumn{2}{|c|}{ Anotar } & \\
\hline 2. Sexo & \multicolumn{2}{|c|}{ ( ) Femenino ( ) Masculino } & \\
\hline 3. Edad & \multicolumn{2}{|c|}{$\begin{array}{l}\text { ( ) } 18 \text { a } 24 \text { años, ( ) } 25 \text { a } 34 \text { años, ( ) } 35 \text { a } 44 \text { años } \\
\text { ( ) } 45 \text { a } 55 \text { años, ( ) más de } 55 \text { años. }\end{array}$} & \\
\hline \multicolumn{4}{|l|}{ 4. Fecha } \\
\hline \multicolumn{4}{|l|}{ 5. Escuela } \\
\hline \multicolumn{2}{|c|}{ Pregunta } & Respuesta & Cód. \\
\hline $\begin{array}{l}\text { 6. ¿Conoce casos de } \\
\text { lar? }\end{array}$ & leserción esco- & ( ) SI ( ) No & \\
\hline \multicolumn{2}{|c|}{$\begin{array}{l}\text { 7. ¿A qué edad cree usted que los ni- } \\
\text { ños y niñas deben de terminar la escue- } \\
\text { la obligatoriamente? }\end{array}$} & & \\
\hline \multicolumn{2}{|c|}{$\begin{array}{l}\text { 8. Podria definir lo que es abandonar la } \\
\text { escuela por parte de los niños. ¿Que } \\
\text { tipo de abandonos existen? }\end{array}$} & & \\
\hline \multicolumn{2}{|c|}{$\begin{array}{l}\text { 9. ¿Cree usted que sus alumnos, alum- } \\
\text { nas y sus padres y madres de familia } \\
\text { son conscientes de los efectos de la } \\
\text { deserción escolar? }\end{array}$} & ( ) Si ( ) No Por qué? & \\
\hline \multicolumn{2}{|c|}{$\begin{array}{l}10 \text { ¿Que tan frecuente es este proble- } \\
\text { ma (deserción y repetición) en su es- } \\
\text { cuela? }\end{array}$} & $\begin{array}{l}\text { ( ) mucho ( ) poco ( ) nada. } \\
\text { Explique }\end{array}$ & \\
\hline \multicolumn{2}{|c|}{$\begin{array}{l}\text { 11. ¿ En su aula qué tan frecuente es la } \\
\text { deserción y repetición escolar? }\end{array}$} & $\begin{array}{l}\text { ( ) mucho ( ) poco ( ) nada. } \\
\text { Explique }\end{array}$ & \\
\hline \multicolumn{2}{|c|}{$\begin{array}{l}\text { 12. ¿Qué hace la institución escolar } \\
\text { donde usted trabaja para evitar la de- } \\
\text { serción escolar? }\end{array}$} & Explique & \\
\hline \multicolumn{2}{|c|}{$\begin{array}{l}\text { 13. ¿Qué hace usted en el aula para } \\
\text { evitar la deserción escolar? }\end{array}$} & Explique & \\
\hline \multicolumn{2}{|c|}{$\begin{array}{l}\text { 14. Anote las principales dificultades } \\
\text { para evitar la deserción escolar. }\end{array}$} & & \\
\hline
\end{tabular}

Muchas gracias por sus respuestas. 
15. Ordene las siguientes causas de deserción escolar según como usted considere que ocurren. Siendo 1 la que afecta más en la deserción y 14 la que afecta menos.

\begin{tabular}{|c|c|}
\hline $\begin{array}{l}\text { ( ) Falta de recursos en el seno familiar pa- } \\
\text { ra mantener los gastos en materiales necesa- } \\
\text { rios (carga económica para la familia) }\end{array}$ & ( ) Problemas emocionales y en el hogar \\
\hline ( ) Reemplazan la escuela por el trabajo & ( ) Uso de drogas \\
\hline ( ) Repiten el grado. & ( ) Metodologia de trabajo en el aula. \\
\hline $\begin{array}{l}\text { ( ) Abandono por incorporación a las labo- } \\
\text { res agrícolas temporales }\end{array}$ & $\begin{array}{l}\text { ( ) Carencia de materiales didácticos ade- } \\
\text { cuados }\end{array}$ \\
\hline $\begin{array}{l}\text { ( ) Labores en el hogar que impide un de- } \\
\text { sarrollo adecuado en las labores de la escue- } \\
\text { la. }\end{array}$ & $\begin{array}{l}\text { ( ) Relación entre el docente con sus } \\
\text { alumnos. }\end{array}$ \\
\hline $\begin{array}{l}\text { ( ) Mal estado de la infraestructura del cen- } \\
\text { tro educativo }\end{array}$ & $\begin{array}{l}\text { ( ) Motivación y participación de los padres } \\
\text { en el proceso educativo }\end{array}$ \\
\hline ) Contenido de los programas educativos & ) Condición migratoria del estudiante \\
\hline
\end{tabular}

Podría agregar otra (as) causa (as) de la deserción escolar a la lista anterior

\begin{tabular}{|c|c|c|}
\hline Pregunta & Respuesta & Cód. \\
\hline $\begin{array}{l}\text { 16. Considera usted que la deserción es una } \\
\text { violación a derecho a la educación. }\end{array}$ & ( ) Si ( ) No & \\
\hline $\begin{array}{l}\text { 17. ¿Promueve usted el derecho a la educa- } \\
\text { ción en sus clases? }\end{array}$ & ( ) Sí ( ) No Explique & \\
\hline $\begin{array}{l}\text { 18. ¿Qué aspectos de nuestra práctica edu- } \\
\text { cativa pueden significar un obstáculo para } \\
\text { que niñas, niños y adolescentes sientan la } \\
\text { necesidad de abandonar la escuela? }\end{array}$ & $\begin{array}{l}\text { Ordene de } 1 \text { en adelante } \\
(\text { ) relación con la maestra (o) } \\
(\text { ) relación con las y los compañeros. } \\
(\text { ) metodología en el aula. } \\
(\text { ) material didáctico. } \\
\text { Otros: }\end{array}$ & \\
\hline $\begin{array}{l}\text { 19. En qué aspectos repercute que los niños } \\
\text { y niñas abandonen la escuela o repitan gra- } \\
\text { dos para el cumplimiento de los derechos de } \\
\text { la niñez. }\end{array}$ & & \\
\hline $\begin{array}{l}\text { 20. ¿Cuál es la importancia que tiene la edu- } \\
\text { cación, para los niños, niñas y adolescentes? }\end{array}$ & & \\
\hline
\end{tabular}

Muchas gracias por sus respuestas. 